\title{
Tratamiento de 37 niños enuréticos con Imipramina
}

DRES.: OSVALDO GASC *, EDECIA OCHOA * y HERNAN DEL PINO R. *.

INTRoDlcción. La imipramina ha venido siendo usada con éxito en el tratamiento de la enuresis desde hacc más de 10 años. Son numerosos los trabajos publicados $(1,2,3,4,5)$ con grupos controles y técnicas de doble ciego que han demostrado su efectividad. Por ser la enuresis una situación frecuente que motiva problemas importantes del desarrollo infantil, tanto en sus aspectos intrafamiliares como en la adaptación a la vida social, y que significa para el médico la necesidad de un enfoque equilibrado entre los aspectos biológicos, psicológicos y sociales, hemos visto con sumo interés la aparición de esta droga y las nuevas perspectivas que nos plantea su uso. Se ha sostenido que esta droga tiene, en dosis farmacológicas, un efecto anticolinérgico, por lo cual su uso permitiría sobrepasar la situación de educsción vesical, que se traduciría en una disminución frecuente de la capacidad vesical del niño enurético (6). Por ser, dentro de los psicofármacos, un psicoestimulante, permitiría modificar la tercera y cuarta etapas de los ciclos del sueño descritos electroencefalográficamente (7), pues frecuentemente la enuresis se produce durante la tercera y cuarta etapas del primero y último ciclo del sueño. Sin embargo, el entrar en el terreno de la psicofarmacología implica la necesidad de un estudio a largo plazo con el fin de observar si el control del síntoma no se encuentra relacionado con la aparición de otros problemas detectados y la necesidad, también, de un estricto control desde el punto de vista orgánico.

MATERLAL Y MÉTODos. Se analizaron las fichas correspondientes a 37 niños enuréticos tratados con imipramina en el consultorio de Nefrología. Los pacientes fueron enviados desde los consuitorios periféricos o desde la policlínica de Psiquiatría. Las edades fluctuaron entre 4 y 15 años, per-

\footnotetext{
* Departamento de Pediatria. Hospital Roberto del Río.
}

TABLA No 1

37 NINOOS ENURETICOS TRATADOS CON IMIPRAMINA. DISTRIBUCION POR EDAD Y SEXO

\begin{tabular}{rcccc}
\hline Edad & Masculino & Femenimo & Total \\
\hline 4 a 7 años & 8 & 5 & 13 \\
8 a $110 \%$ & 8 & 11 & 19 \\
12 a 15 r & 1 & 4 & 5 \\
\hline T o t a 1 & 17 & 20 & 37 \\
\hline
\end{tabular}

teneciendo todos a un similar nivel socio-económico. (Tabla 1). Se tomó una historia médica personal y familiar con especial referencia a su motivo de consulta. Todos los niños se orinaban duráte el sueño desde el nacimiento, salvo 4 niños que habían controlado el esfínter vesical en algún momento y por períodos variables, pero que estaban humedeciendo la cama de nuevo desde por lo menos un año antes de consultar. A todos se les hizo examen de orina completo, urocultivos se riados y en ocho casos se practicó pielografía, siendo todos normales desde el punto de vista nefrourológico. No se consideraron para este estudio los enuréticos infrecuentes (períodos secos alternando con períodos húmedos). También se rechazaron los niños enuréticos tratados y que presentaban retordo mental o signos de pielonefritis u otro factor orgánico que pudiera explicar la enuresis. Ningún nião tenía epilepsia.

De acuerdo con los antecedentes de trabajos anteriores, se informó a los padres sobre los probables efectos adversos de la droga y sobre el tiempo que ésta demoraría en hacer efecto. Se les citó cada 15 días para discutir los resultados y posibles efectos no deseables. De acuerdo con la respuesta obtenida se hacía los ajustes de dosifi- 
cación y si había buena respuesta se citaba al mes siguiente.

A los niños menores de 7 años se comenzó administrándoles una dosis de $10 \mathrm{mgr}$. de imiprimina; hasta 12 años se les dio $25 \mathrm{mgr}$ y a los mayores de 12 años se les proporcionó una dosis de $50 \mathrm{mgr}$ del medicomento diaria. Esta dosis se daba media a una hora antes de acostarse y una vez obtenida la respuesta esperada, se prolongó el tratamiento durante dos meses. Los niños que no respondieron a la dosis inicial recibieron cantidades mayores: $25 \mathrm{mgr}$ los menores de 7 años, $50 \mathrm{mgr}$. los menores de 12 años y $75 \mathrm{mgr}$ los mayores de 12 años.

El medicamento fue entregado por la farmacia del hospital y en algunas oportunidades fue adqujrido en farmacias comerciales.

Además de conversar con el niño y los padres sobre los probables buenos resultados que se obtendrían, se les recomendó que redujeran la ingestión de líquidos a fartir de las 18 horas, ofreciéndosele en la noche una comida con contenido acuoso restringido.

Dado que la droga empleada poseía antecedentes claros de tener un efecto farmacológico sobre la enuresis, no se consideró la posibilidad de utilizarlo en un grupo control. El efecto placebo tampoco se empleó por disponerse de información previa. E1 médico investigador (O. G.) no cambió durante toda la experiencia.

Resultados. Distribuimos los niños en 3 grupos de acuerdo con la dosis dcl medicamento que recibieron según las edades, comprobándose que la gran mayoría se ubica por debajo de los 12 años.

En nuestra casuística se observó una cifra mayor de mujeres que hombres. Esta diferencia se hace evidente a medida que se avanza en la edad.

En el primer control, luego de iniciada la administración de imipramina, es decir, a las 2 semanas, 24 niños del total de $37(66 \%)$ amanecian secos, por lo que se les mantuvo la dosis inicial hasta completar 2 meses de tratamiento. De los 13 niños que no respondieron inicialmente, 7 tuvieron una buena respuesta al aumentar la dosis primitiva del medicamento elevando el total de buenas respuestas a 31 pacientes, es decir, $84 \%$ de éxito. (Tabla 2).

Seis niños $(16 \%)$ no experimentaron ninguna mejoría aún después de haber elevado la dosis a los niveles máximos para cada grupo, debiendo suspenderse la droga luego de varias semanas de tratamiento.

Del grupo de 13 niños menores de 7 años, 8 respondieron satisfactoriamente a la dosis de 10 mgr diarios ya en el primer control. A los 5 restantes se les aumentó la dosis a $25 \mathrm{mgr}$ obtenién. dose una buena respuesta en 3 de ellos. Todos
TABLA Nọ 2

37 NINOS ENURETICOS TRATADOS CON IMIPRAMINA, RESPUESTA AL TRATAMIENTO

\begin{tabular}{|c|c|c|c|c|c|c|}
\hline \multirow{2}{*}{ Grupos } & \multicolumn{6}{|c|}{ Antes dia 15 Después dia IS Resp. final } \\
\hline & Ruena & Mala & Bueno & Mala & Buena & Molat \\
\hline 4 a 7 años & 8 & 5 & 3 & 2 & $1 \mathrm{I}$ & 2 \\
\hline 8 a 11 & 13 & 6 & 3 & 3 & 16 & 3 \\
\hline 12 a 15 & 3 & 2 & 1 & 1 & 4 & 1 \\
\hline $\mathrm{T}$ o t a I & 24 & 13 & 7 & 6 & 31 & 6 \\
\hline
\end{tabular}

cumplicron 2 meses de tratamiento con la dosis efectiva, una vez superado el problema. Ninguno manifestó signos importantes que determinaran. suspender la droga, presentándose una cierta irritabilidad en 5 nin̄os ( 2 con $25 \mathrm{mgr}$. y 3 pacientes con $10 \mathrm{mgr}$ ).

En el grupo de 19 niños, cuyas edades fluctuaban entre 8 y 11 años, 13 pacientes no se orinaban durante el sueno luego de recibir 25 mgr. diarios de imipramina durante 15 días. A los 6 niños que no respondieron se les aumentó la dosis a $50 \mathrm{mgr}$, respondiendo esta vez bien 3 de ellos. A los otros 3 pacientes se les administró $75 \mathrm{mgr}$. diarios, no obteniéndose respuesta favorable y manifestando 2 de ellos gran nerviosismo, sueño in. tranquilo y dolor abdominal, por lo que hubo de suspenderse esta medicación.

En el grupo de niños mayores de 12 años, se obtuvo una buena e inmediata respuesta en 3 de los 5 pacientes. A los 2 niños que no respondieron se lcs aumentó a 75 mgr. al día, obteniéndose la respuesta esperada en 1 de los dos. En este grupo no hubo efectos laterales del medicamento.

Segulmiento. Los nin̆os que respondieron satisfactoriamente se mantuvieron así hasta por lo menos un año de suspendido el tratamiento, salvo en 7 casos, que volvieron a presentar el síntoma algunos meses despućs de terminado el primer ensayo terapéutico. En estos casos una nueva cura con la misma dosis recibida antes, determinó una buena respuesta rápidamente, haciéndose en estos pacientes una terapia más reducida en el tiempo, aproximadamente de un mes. No se han detectado problemas nefro-urológicos ni conductuales hasta el momento de esta evaluación.

Discusión. Dentro de las condiciones de la experimentación clínica desarrollada, consideramos que la imipramina es una droga, en primer lugar, con un efecto farmacológico evidente sobre el síntoma en estudio. Si bien, por las razones que hemos dado anteriormente, no incorporamos a nuestra experiencia el uso del placebo para analizar el 
efecto psicológico aislado de la acción terapéutica empleada, estudios anteriores $(1,3)$ nos permiten sustentar que no cs esta la razón del efecto observado.

Cuando el síntoma estudiado se modifica por la droga empleada, estc efecto se ve fundamentalmente en el período de los primeros 15 días $(66 \%)$. Si bien es cierto que el aumentar la dosis $y$ el tiempo de tratamiento aumenta el porcentaje de éxito, ya este efecto no es tan relevante como en el período anterior y con dosis más bajas.

El efecto del tratamiento persiste después de la suspensión de la droga, siendo el porcentaje de recaídas de $22 \%$. Sin embargo, estas recaídas responden a las mismas dosis y en iguales tiempos, uniformamente bien, siendo seguidas de un período de desaparición del síntoma.

El control del síntoma motivó una reducción de la angustia de los padres en relación al síntoma del hijo, una normalización de la vida familiar que habia sido alterada por la enuresis.

El efecto psicofarmacológico no se vio acompañado de trstornos evidentes de la vida escolar ni de estimulación en otras áreas de la conducta infantil.

Sin embargo, serán necesarias nuevas investigaciones para aclarar los efectos a más largo plazo.

Por último, llama la atención en nuestra casuística la distribución semejante entre hombres y mujeres, pues en la bibliogratía revisada (1) aparece la enuresis como un problema significativamente más frecuente en el sexo masculino. En nuestro material la preponderancin del sexo femenino se acentúa a mayor edad, lo que nos hace pensar que el síntoma enuresis es sometido en nuestra sociedad a mayor control en los hombres a medida que la edad aumenta, lo cual abre una interesante posibilidad acerca de la real frecuencia del síntoma enuresis en el varón en Chile y la posibilidad de que éste consulte a nuestro sistema de salud.

\section{RESUMEN}

Se analiza la respuesta inmediata y a largo plazo de 37 niños enuréticos (edad 4 a 15 años), tratados con imipramina.

No hubo diferencia significativa en cuanto $a$ distribución por sexo.

Las dosis de imipramina oscilaron entre $10 y$ 75 mgr. diarios y fueron dadas durante 8 semanas.

$A$ las dos semanas el $66 \%$ de los niños amanecian secos y al cabo de cuatro semanas, luego de ajustar la dosis del medicamento, se totalizó un $84 \%$ de buenas respuestas.

6 niños (16\%) no mostraron ninguna mejoría, aun cuando la dosis alcanzó los máximos niveles.
Los 31 niños que respondieron satisfactoriamente han sido seguidos por más de un año, observándose recaida en 7 de ellos (22\%), los que luego de una nueva cura dejaron de humedecer la cama. No se han constatado trastornos conductuales ni orgánicos durante el seguimiento.

\section{SUMMARY}

The inmediate and long-term response of thirty seven outpatients enuretic children (aged 4 to 15 years), treated with imipramine are analized.

There wasr't any significant difference with regard to sex distribution.

The dosage of imipramine oscillated between 10 to $75 \mathrm{mgr}$. daily for a period of eight weeks.

After two weeks 66 per cent of the children becam: dry and in four weeks, after the medicine dose regulaton, 84 per cent of good responses were added up.

Six children ( 16 per cent) did not show any impro. vernent, even when the dosa was raised to the maximum levels.

Thirty one children that had a good response were followed for more than a year, relapse was observed in 7 of them (22 per cent) who after a new cure stopped the bedwetting.

During the follow-up study we have not observed neither behavior nor organic changes.

\section{REFERENCIAS}

1.-Miller $P . R$., et al. Imipramine in the treatment of enuretic schoolchildren. A double blind study. Amer. J. Dis. Child. 115: 17-20, 1968.

2.-Fridloy $G$. A., $c t$. al. Treatment of enuretic chikdren with imipramine (Tofranil). Clin. Pediat. (Phila) 5: $175-176,1966$.

3.-Poussaint A. F, et al. A controlled study of imipramine (Tofranil) in the treatment of child rood enuresis. J. Pediat. 67: 283-290, 1965.

d.-Cahill, C. A. Imipramine in nocturnal enuresis. Amer. J. Dis. Child 114: 215, 1967.

5.-Epstein S. J., et. al. Imipramine (Tofranil) in the control of enuresis. Aner. J. Dis. Child. 109: 412$415,1965$.

6.- Muether $S . R$. Development of urinary control in children: a new concept in cause, prevention, and trcatment of primary enuresis. J. Urol. 84: 714 , 1960.

7.-Hartmans, $E$. On the pharmacology of dreaming sleep (ihe D. State). J. Nerv, Mental Dis. 146: $165.173,1968$.

8.-Muellner $S$. $R$. The physiology of micturition. J. Urol. 65: 805, 1951.

9.-Muellner $S$. $R$. The voluntary control of micturition in man. J. Urol. 80: 473, 1958. 